\title{
Using general public connected devices for disasters victims location
}

\author{
Olivier Sébastien, Fanilo Harivelo, Didier Sébastien
}

\begin{abstract}
Laboratoire d'Informatique et de Mathématiques - Université de la Réunion (France) \{olivier.sebastien,
\end{abstract} fanilo.harivelo, didier.sebastien\}@univ-reunion.fr

\begin{abstract}
Information Technologies (IT) have largely contributed to improve disaster management by providing new means to acquire, process and broadcast information to the various actors. However, most of the work was targeted toward the risk experts: decision makers, rescue teams, firemen, etc.

Nowadays, a new parallel trend seems to emerge: it consists in taking into account signal from consumer devices like cell phones (basic ones to smartphones), tablets, computers or other devices but light networks equipment (routers, WiFi access points) too. More particularly, our goal is to rely on the wave activity generated by those equipments, which are generally personal, to detect where victims are more likely to be located after a disaster, typically a seism. activity.

To reach this aim, we present the feasibility of mixing two approaches to acquire data by measuring their radio

\section{Introduction}

The ten last years saw the dramatic increase of wireless communication and mobile personal devices, that is to say digital terminals that are attached to one person rather than a place (like a home or office phone): mobile phone of course (including smartphones), tablets, laptops, etc. This brought a new opportunity in risk management that can be summed up briefly: if one of these devices is detected in a place where a disaster occurred, there are a lot of chances that there might be people to rescue in that particular place.

Thus, the question is to define ways of identification of such mobile terminals, according to the various technologies they embed. Indeed, most advanced ones provide lots of possibilities to retrieve their users location, such as Android-powered smartphones, which can track and report continuously to the Google Location History service all the places they went [1]. But this approach might not be sufficient because not everyone owns such terminals and of course because of privacy issues.
\end{abstract}

\section{Detection process overview}

The proposal consists in relying on autonomous equipment such as drones or robots [2] to roam a disaster scene in order to draw a map of possible victims location when no communication infrastructure remains active, as shown in Figure 1. In fact, the idea is more to guide the rescue team to place where chances are higher to find a survivor than to locate him with accuracy and certitude. This approach is complementary to the existing ones and does not aim at replacing them.

To achieve this, the autonomous equipment is fitted with antennas which specifications will be discussed thereafter. It is sent on the terrain looking for response to its broadcast requests. Once a response is detected, identification is attempted to characterize it and finally the GPS position along with all the metadata (time, signal strength, network type...) is sent to the local command centre. The latter thus having information to take appropriate decisions.

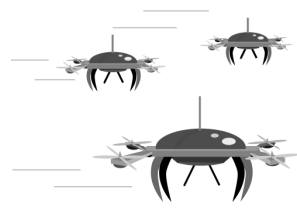

1. Drones fleet is sent over a disaster area

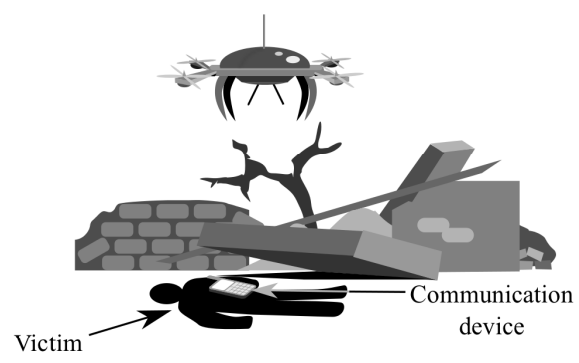

2. Victim is stuck under the debris, with a communication device with him/her
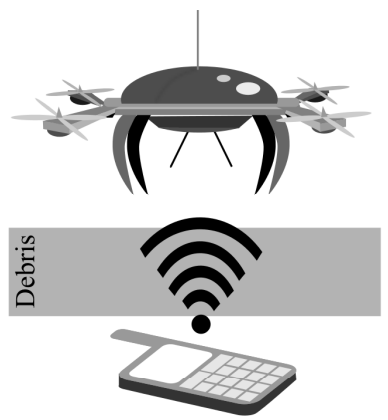

3. The drone simulates the normal infrastructure so that the communication device initializes the "shake hands" procedure. It is thus detected.

Figure 1: overview of the detection principle 
Let us focus on the detection part. We are particularly interested in the initial response a connected device gives. Actually, most of them are designed to look for a network medium as soon as they are powered and to perform continuously this search while a connection is not established.

\section{Devices characterization}

The principle shown before is possible because the general public now owns a lot of digital wireless devices. Those terminals may be very different in term of usage of course but in term of networking specifications too. That is why we need to list them along with their connectivity.

\subsection{Connected objects}

Connected objects are more and more common in nowadays society. They consist in very different shaped useful tools to communicate among users or simply ease actual processes. Table 1 presents the main devices and the features relevant to our subject. Most of them can be linked to the presence of at least a single user, regarded to the service they are providing.

Table 1

\begin{tabular}{|c|c|c|c|c|c|c|c|c|}
\hline & & & & & & & & \\
\hline & laptop & $\begin{array}{l}\text { mobile } \\
\text { phone }\end{array}$ & phone & smart watch & $\begin{array}{l}\text { activity } \\
\text { tracker }\end{array}$ & ear-flap & $\begin{array}{c}\text { wireless } \\
\text { mouse }\end{array}$ & gamepad \\
\hline Service & various & various & talk remotely & $\begin{array}{l}\text { connection } \\
\text { with } \\
\text { smartphone }\end{array}$ & log moves & $\begin{array}{l}\text { connection } \\
\text { with } \\
\text { smartphone }\end{array}$ & $\begin{array}{c}\text { computer } \\
\text { control }\end{array}$ & $\begin{array}{l}\text { console } \\
\text { control }\end{array}$ \\
\hline Usage & office/home & office/home & office/home & home & office/home & office & office/home & home \\
\hline $\begin{array}{c}\text { Wireless } \\
\text { Technology }\end{array}$ & $\begin{array}{c}\text { wifi, } \\
\text { bluetooth }\end{array}$ & $\begin{array}{l}3 \mathrm{G}, \text { wifi, } \\
\text { bluetooth }\end{array}$ & DECT/GAP & bluetooth & bluetooth & bluetooth & bluetooth & bluetooth \\
\hline
\end{tabular}

As humans tend to live in groups, a living space is susceptible to emit a wide spectrum of signals. That is why it is relevant to characterize them quantitatively and qualitatively. The identification of areas emitting a large range of signals from several devices can be a great key to guide rescuers. Of course, as it will be detailed in next part, waves propagation is very different. Thus, several steps could be defined to focus first on low frequency waves, then refine the received information by tracking shorter range signals. Qualitatively, the signal can be characterized by the kind of service offered to define the type of device. For instance the service offered by an ear-flap is more strongly linked to its user than a laptop. That is why it would be more relevant to prioritize researches where this type of device is detected.

\subsection{Networks}

We present hereafter (Table 2) the commonly available wireless technologies in mobile devices:

Table 2

\begin{tabular}{|c|c|c|c|c|}
\cline { 2 - 4 } \multicolumn{1}{c|}{} & $\begin{array}{c}\text { Bluetooth v2.0 EDR } \\
\text { (class 2) }\end{array}$ & WiFi (IEEE 802.11g) & GMM \\
\hline Frequency Band & $2.4 \mathrm{GHz}$ & $2.4 \mathrm{GHz}$ & $900 \mathrm{MHz} / 1800 \mathrm{MHz}$ & $2 \mathrm{kbits} / \mathrm{s}$ \\
\hline Max. data rate & $3 \mathrm{Mbits} / \mathrm{s}$ & $54 \mathrm{Mbits} / \mathrm{s}$ & $2 \mathrm{Mbits} / \mathrm{s}$ \\
\hline $\begin{array}{c}\text { Max. output power of } \\
\text { the handset }\end{array}$ & $2.5 \mathrm{~mW}(4 \mathrm{dBm})$ & $100 \mathrm{~mW}(20 \mathrm{dBm})$ & $\begin{array}{c}1 \mathrm{~W}(30 \mathrm{dBm}) \mathrm{for} 1800 \\
\mathrm{MHz} \text { and } 2 \mathrm{~W}(33 \mathrm{dBm}) \\
\text { for } 900 \mathrm{MHz}\end{array}$ & $\begin{array}{c}125 \mathrm{~mW}(21 \mathrm{dBm})-251 \mathrm{~mW}(24 \\
\mathrm{dBm})\end{array}$ \\
\hline Max. range & $10 \mathrm{~m}$ & $\begin{array}{c}\text { Approx. } 38 \mathrm{~m} \text { (interior) } \\
\text { Approx. } 140 \mathrm{~m} \\
\text { (exterior) }\end{array}$ & $35 \mathrm{~km}$ \\
\hline
\end{tabular}

While Bluetooth exposes interesting feature related to low energy consumption, its short transmission range and weak radiated power limit its use in disaster recovery. WiFi and GSM/UMTS suit best emergency situation in which a robust search and rescue network (RSN) must be quickly operational. Indeed, the potential presence of obstacles coupled with the blind search and scan of victims and survivors impose a high penetration capability of the transmission despite signal attenuation and interferences. Mentioned technologies have proven efficiency in similar situations. Portable Disaster Recovery Network [3] introduces a network architecture composed of one or more Access Points, Gateway node, DRN Phones dropped from an helicopter and on-site Command Center. Communications use standard wireless and mobile technology such as WiMAX, WiFi, 3G, 2G, or $4 \mathrm{G}$.

Another area pertaining the positioning or location of individuals or objects using WiFi is Device-free Passive (DfP) [4] localization. It consists in detecting, identifying and tracking entities that do not carry any device by exploiting indoor WiFi network. A radio map is constructed initially. Then, changes in the environment are detected by monitoring and processing received signals. Indeed, people and entities disturb the pattern of radio waves. Radio planning in mobile networks has to consider obstacles, building and wall traversal as the main part of the mobile traffic is indoors [5]. The 
base station and the handset perform well despite the varying wireless conditions (attenuation, signal absorption, interferences, ...) thanks to power control. The nature and characteristics of the material in-between, are factors that determine those conditions. For example, authors of [6] study the effects of the presence of multiple reinforced concrete walls on the shielding effectiveness in GSM and UMTS systems. Two wall configurations are considered: a room between two $0.35 \mathrm{~m}$-width reinforced concrete walls and a room between a $0.35 \mathrm{~m}$-width ceiling and a perfectly conductive floor. An average attenuation of $5 \mathrm{~dB}$ has been found. Thus, the communication is still feasible. While a WiFi network is easily deployable GSM/UMTS-compatible devices are more widespread (the are currently about 3 billions phones). On the other hand, GSM and UMTS requires the support of a heavy infrastructure. The coverage of the whole disaster terrain may be too costly and time-consuming to be feasible within a few hours after the disaster event (more than $50 \%$ of deaths occurred in this period [3]). We suggest a combined approach: the deployment of both small scale and localized WiFi networks and GSM/UMTS network.

\section{Terrain behaviour}

One cannot stay at the theoretical normative aspects because in real conditions, various non-controllable aspects must be taken into account. Therefore our goal in this part is to deal with the constraints that can be encountered and the way to deal with them.

\subsection{WiFi networks cartography concerns}

First of all, the WiFi networks cartography is an operation that must be accomplished before a disaster occur. There is a lack of strategy concerning this point: first of all, it raises legal issues concerning privacy protection and then it is difficult to determine who should be in charge of this action, either public authorities or private companies [7]. Technically speaking, many solutions exist to achieve the mapping. Of course, the goal is to make the process the more automatic possible. Once again, our proposal consists in relying on autonomous drones, as defined in [8]. They are now able to crawl indoor [2] and provided they are equipped with the right interfaces, they can easily map the buildings.

WiFi mapping also raises the question of the efficiency of the pre/post-disaster comparison. Lots of work has already been done in this field [9], for instance by studying automatically the difference between satellite pictures of an area taken before and after a crisis. The main issue is the reliability of the interpretation: there are reasons for the environment to change without implying the occurrence of a disaster. Unless if updates are made regularly, and under the supervision of a human control it is difficult to ensure that the process will deliver a high rate of detection. There are many ways to address this context. The first one consists in multiplying the number of network devices. We are in a favourable context as this is the case since Wireless Local Area Networks (WLAN) have started to become common a decade ago. The second trend is related to the tasks that can be automated by the devices themselves: since they are getting smarter, the cartographic update can be scheduled and sent to a server located far from the area, which is a requisite in any situation.

\subsection{GSM/UMTS network scan concerns}

Lots of parameters can alter the mobile network scan as evoked by [10] and [11] in the context of the I-LOV project. Most of them are related to the energy consumption of the whole process in general and of the devices on the terrain in particular. Indeed, battery life is a major issue, especially for smartphones, which tend to have a more or less 24 hours autonomy, given all the features they have. The solution here depends on the manufacturers policy. They tend to implement energy-saving features, which disable dynamically some interfaces when they are not used. Therefore, situation should be better in a near future.

Moreover, we made the hypothesis that no fixed infrastructure was available after the disaster. This allows the area to be clear of "pollution" from a radio point of view. We believe this is mostly the case when a major crisis occurs. But we must keep in mind that if the infrastructure remains active, public authorities along with network carriers have to deal with this particular point, either by shutting it down selectively or by using jamming devices as exposed by [11].

From the drone/robot crawling perspective, recent research [2] proved that the autonomous navigation should not be an issue. However, what need to be assessed is the precision of the measurement, especially as far as the geographical positioning is concerned. Indeed, Global Navigation Satellite System (GNSS) is the main resource that may be used. This raises first the question of the selection of the best system (GPS, Glonass, ...) for a given place and then the lack of local triangulation assistance can limit the accuracy. A possible solution for this particular point consists in adding an inertial system to the drone/robot and to rely on a synchronised evolution of a fleet instead of an individual device. Thus, each drone/robot is able so compare its measured position to the one estimated from the other ones crawling in the neighbourhood. 


\section{Conclusion}

The goal of this article was to present a contribution in the detection of victims after a disaster by relying on popular connected devices. They have the advantage to be very common and always located on the terrain. Our proposal blends two approaches that can exist separately but which may dramatically ease victims rescue by allowing a faster response time and by restricting the area where to operate.

The next step consists in leading terrain experimentations to acquire data to validate the approach. Indeed, we were not able for the moment to anticipate some behaviours. For instance, a typical mobile phone is supposed to look for a network while none is detected. This search requires the device to use full power, which drain the battery a lot. That is why some manufacturers may decide of a time out to spare it. We thus have to check if this kind of policy is still compliant to the aim we try to reach.

Another aspect concerns the adaptation of the approach considering the geographical area. Of course, it has reasonable chance to work in lots of places with an average standard of life. However, disasters can strike everywhere. So, the question is to determine how such a system can behave in developing countries with emerging networks infrastructure.

\section{References}

1. https://support.google.com/gmm/answer/3118687?hl=en consulted 14/02/14

2. L. Aprille, J.L. Dugelay, B. Ranft, « Indoor Autonomous Navigation of Low-Cost MAVs Using Landmarks and 3D Perception ", in Proceedings of OCOSS'2013: Ocean \& Coastal Observation: Sensors and observing systems, numerical models \& information. October 2013, pp 307-312.

3. Ram G. Lakshmi Narayanan and Oliver C. Ibe, «A joint network for disaster recovery and search and rescue operations », in Computer Networks. 56, 14, 2012, 3347-3373.

4. M. Moussa and M. Youssef, "Smart Devices for Smart Environments: Device-free Passive Detection in Real Environments", IEEE International Conference on Pervasive Computing and Communications, Galveston, TX, 2009, pp. $1-6$.

5. M. Tolstrup, Indoor Radio Planning: A Practical Guide for GSM, DCS, UMTS and HSPA, Wiley, 2008.

6. G. Antonini, A. Orlandi, S. D’Delia, "Shielding Effects of Reinforced Concrete Structures to Electromagnetic Fields due to GSM and UMTS Systems”, IEEE Transactions On Magnetics, Vol. 39, No. 3, May 2003.

7. Marc J. Blitz, "The Right to Map (and Avoid Being Mapped): Reconceiving First Amendment Protection for Information-Gathering in the Age of Geotagging and Google Earth." 14 Columbia Science and Technology Law Review 115-202 14 (2013): 115-202.

8. W. Wang, R. Joshi, A. Kulkarni, W. K. Leong, B. Leong, «Feasibility study of mobile phone WiFi detection in aerial search and rescue operations », in Proceedings of the 4th Asia-Pacific Workshop on Systems (p. 7). ACM.

9. I. Kyrgyzov, M. Campedel, M. Roux, T. Tanzi, S. Rital, Final Report EXITER 08. Technical report, 2008.

10. S. Zorn, R. Rose, A. Goetz, R. Weigel, « A novel technique for mobile phone localization for search and rescue applications », in Indoor Positioning and Indoor Navigation (IPIN), 2010 International Conference on (pp. 1-4). IEEE.

11. S. Zorn, M. Maser, A. Goetz, R. Rose, R. Weigel, « A power saving jamming system for E-GSM900 and DCS1800 cellular phone networks for search and rescue applications » in Wireless Sensors and Sensor Networks (WiSNet), 2011 IEEE Topical Conference on (pp. 33-36). IEEE. 